\title{
Use of Tenckhoff Catheter for Peritoneal Dialysis in Terminal Renal Failure
}

\author{
P. G. LANKISCH, \\ H. J. TÖNNIS, \\ E. FERNANDEZ-REDO, J. GIRNDT, \\ P. KRAMER, \\ E. QUELLHORST, F. SCHELER
}

secured with sutures in the subfascia. The middle section of the catheter was brought through a subcutaneous tunnel and the second cuff positioned in the tissues immediately beneath the skin at the point of emergence of the catheter. The external section

\section{Summary}

Over a period of 33 months a total of 2,146 peritoneal dialyses were carried out by means of indwelling Tenckhoff catheters in 65 patients suffering from terminal renal failure. The patients were maintained on peritoneal dialysis for periods varying from two weeks to 13 months. Treatment over long periods was possible in only a few cases. Infection and clotting, which tended to limit the functional life of the catheters, was reduced by rigid asepsis and by adding heparin to the dialysate. The Tenckhoff catheter was found to be valuable for peritoneal dialysis as a short-term measure, especially in patients in whom haemodialysis was not immediately feasible, in borderline cases when kidney function was not too seriously impaired, and as an alternative to haemodialysis when that was interrupted by complications.

\section{Introduction}

Tenckhoff and Schechter's (1968) modified indwelling catheter for peritoneal dialysis differs from the Palmer-Quinton catheter (Palmer et al., 1964, 1966) in having either one or two Dacron felt cuffs for better anchorage and for preventing infection. Dacron has been found to be inert in body tissue (McDonald et al., 1968; Tenckhoff and Schechter, 1968). Despite some earlier favourable reports (Tenckhoff and Schechter, 1968; Tenckhoff and Curtis, 1970; Brewer et al., 1972) the Tenckhoff catheter has still not been widely adopted, and the purpose a of this paper is to record our experience of its use for peritoneal dialysis in patients with terminal renal failure.

\section{Patients and Methods}

During a period of 33 months, from January 1970 to the end of September 1972, a total of 82 Tenckhoff catheters were used in 65 patients. In all, 2,146 dialyses were carried out and the periods for which patients were maintained on treatment ranged from two weeks to 13 months. The sum of the periods of treatment amounted to 262 months.

In most cases the two-cuff Tenckhoff catheter was used. The intra-abdominal section of the catheter was inserted through a midline incision into the pelvic peritoneum and the first cuff

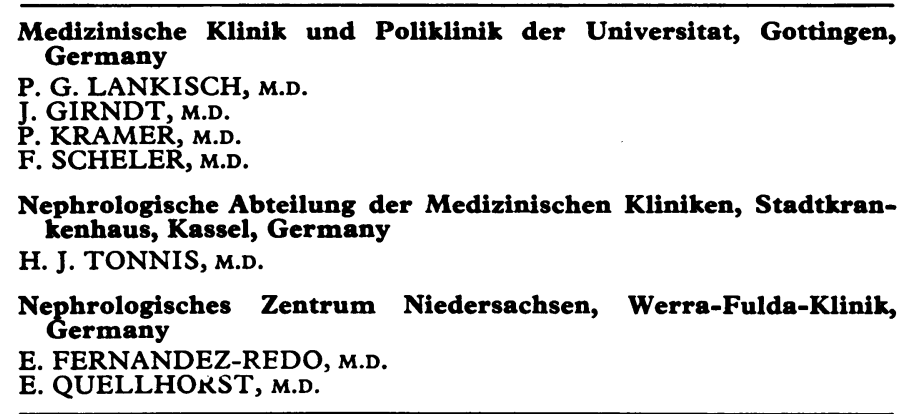
of the catheter was then sealed off and a sterile dressing applied. The first dialysis was begun at once to obviate clotting. Subsequently dialyses for periods of 24 to 36 hours were carried out twice weekly. Heparin 2,500 U/l. was added to the dialysate, and after each dialysis one litre of dialysate was left in the peritoneal cavity to prevent clotting. Samples were cultured weekly to check for infection, care being taken to avoid contamination by organisms colonizing the external end of the catheter.

\section{Results}

By the end of the 33-month period 15 of the 65 patients had died of their primary disease or of complications unconnected with the management of the dialysis; 28 patients had been transferred to haemodialysis (previously planned in 18 cases, and owing to complications of peritoneal dialysis in 10); four patients had received kidney transplants; one patient had been returned to peritoneal dialysis by the repeated puncture technique; and 17 patients were still being maintained by means of the Tenckhoff catheter.

Signs of peritonitis (abdominal pain persisting for more than 12 hours, a rectal temperature of over $38^{\circ} \mathrm{C}$, and clouding of the dialysate) were observed in 21 patients. In three of them the dialysate culture was sterile. In 11 the catheter clogged and had to be removed, but in the remaining 10 cases the peritonitis was effectively treated with the addition of antibiotics to the dialysate

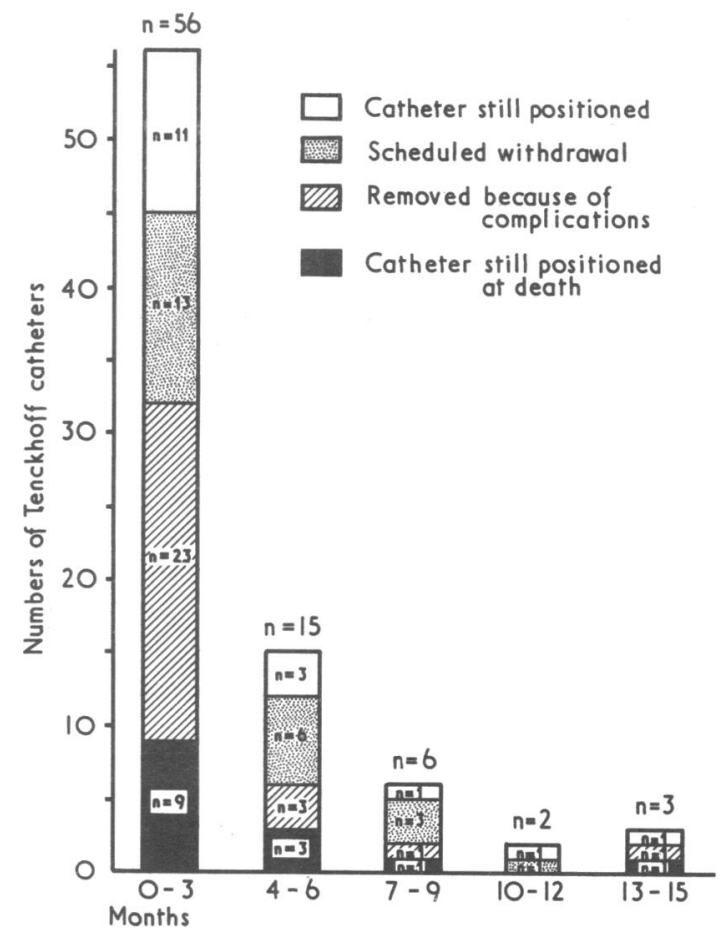

Survival time of 82 Tenckhoff catheters. 
and the catheter continued to function. In three cases without peritonitis the catheter was blocked by a fibrin clot, which periodic instillation of highly heparinized infusions failed to move. In three cases infection developed in the subcutaneous tunnel, and in one of the three an abscess formed. In one case the catheter became dislodged and in another it perforated the intestine.

Weekly cultures of the residual intraperitoneal dialysate showed pathogenic organisms in 58 out of 638 examinations $(9 \cdot 1 \%)$. In 18 cases with a positive culture only peritonitis was found clinically. The organisms most often found were enterococci, Escherichia coli, and staphylococci. The appropriate antibiotics were added to the dialysate in these cases.

The average survival time of the catheters was just over three months (see chart). In a few cases it was more than a year. Most of the patients underwent dialysis for periods of up to six months, and during that time complications (usually peritonitis) were the main reason for catheter changing.

Necropsy was possible in only six of the 15 patients who died. One had an isolated pelvic abscess, and another adhesions of the sigmoid mesentery. Three patients had no adhesions and the catheter lay free in the pelvis.

\section{Discussion}

Peritonitis presented the main problem in the patients in this series. It occurred in almost a third of the cases and in some cases recurrently, ending the functional life of the catheter. A leucocytosis was seldom seen. Tenckhoff and Curtis (1970) reported peritonitis in $13(68 \%)$ out of a total of 19 patients, and Brewer et al. (1972) reported it in $12(50 \%)$ out of 24 patients, with one death. In nine of the 12 the infection was contained by antibiotics, and in only three cases had the catheter to be removed. In comparison, $19(83 \%)$ out of 23 patients dialysed through a Palmer-Quinton catheter developed peritonitis, though only after an average catheter survival of 10.6 months (Palmer, 1971). The proportion of cases of peritonitis was similar in 46 patients in whom a total of 1,476 peritoneal dialyses were carried out by the repeated puncture technique, and it was remarkable that the infection most often occurred between the eighth and ninth month (Quellhorst et al., 1973).

Blocking of the Catheter by fibrin clot, a recognized somplication, rarely occurred in the present series of cases after heparin was added routinely to the dialysate. Infection in the subcutaneous tunnel occurred less often when asepsis was more rigidly observed, and the number of positive cultures of the dialysate also decreased.

The typical complications of dialysis by the repeated puncture technique, such as intestinal, bladder, or vascular injury, did not occur in our series with the Tenckhoff catheter, and we see this as one of its major advantages. Simkin and Wright (1968), on the other hand, reported five cases of intestinal perforation among 443 catheter insertions, three of which were fatal. Kahn et al. (1973) reported three intestinal perforations, one fatal, among 400 peritoneal dialyses by the repeated puncture technique. Finally, among 1,476 catheter insertions Quellhorst et al. (1973) observed one case of intestinal perforation; one of bladder perforation; and two of vascular injury, one fatal.

Patients suffered little discomfort from the indwelling catheter and they were spared the distress of the repeated puncture technique. Also there were few problems of medical or nursing care. The Tenckhoff catheter was originally conceived for use in home peritoneal dialysis, and this should be possible in suitable cases. Only one of our patients was considered suitable, but she had to return to the dialysis centre after some weeks because of peritonitis.

The average functional life of the catheters in our cases was comparable to that in the series of Brewer et al. (1972). Tenckhoff and Curtis (1970), however, reported a serviceable use of up to 28 months. Palmer (1971) was able to dialyse several patients for up to four years with the Palmer-Quinton catheter.

\section{Conclusion}

From our experience of the present series of cases we concluded that peritoneal dialysis with the Tenckhoff catheter is of value as a short-term treatment for terminal renal failure in the following cases: (1) when for lack of an adequately formed arteriovenous fistula or for some other reason haemodialysis is not immediately feasible; (2) when kidney transplantation is to be performed in the near future; (3) in borderline renal failure when the glomerular filtration rate is $5-10 \mathrm{ml} / \mathrm{min}$ and the choice lies between conservative treatment and dialysis; and (4) when haemodialysis is interrupted by complications such as pericarditis or a thrombosed or infected fistula. Long-term treatment is contraindicated except in cases where vascular access is impossible or in some children up to the age of puberty.

Using peritoneal dialysis while waiting for a fistula to open up for haemodialysis may result in clotting in the forming fistula owing to temporary dehydration. This tends to happen in patients who still have a high residual urine output, but in our experience it can best be prevented by intensive care and maintaining a correct fluid balance. The alternative to peritoneal dialysis in this situation is to insert a shunt into one limb and a fistula into another. This has the disadvantage, however, of losing a cannulation site which may be urgently needed later on.

To prevent infection, to avoid clot formation, and to prolong the life of the catheter the following measures are essential: (1) catheter asepsis must be strictly maintained; (2) dialysis must be carried out for a period of 24-36 hours twice weekly, and heparin $2,500 \mathrm{U} / 1$. added to the dialysate; (3) a residuum of one litre of dialysate should be left in the peritoneal cavity after each dialysis; (4) appropriate antibiotics should be added to the dialysate when infection is suspected; (5) samples of the dialysate should be cultured weekly and smears from the catheter inlet cultured when indicated; and (6) suspected displacements of the catheter or impairments of drainage should be monitored by $x$-ray examination.

This paper is dedicated to Professor W. Tönnis, M.D., on the occasion of his 75th birthday.

\section{References}

Brewer, T. E., Caldwell, F. T., Patterson, R. M., and Flanigan, W. J. (1972). Fournal of the American Medical Association, 219, 1011

Kahn, S. I., Garella, S., and Chazan, J. A. (1973). Surgery, Gynecology and Obstetrics, 136, 40.

McDonald, H. P., et al. (1968). Transactions of the American Society for Artificial Internal Organs, 14, 176.

Palmer, R. A. (1971). Canadian Medical Association fournal, 105, 376.

Palmer, R. A., Quinton, W. E., and Gray, J. E. (1964). Lancet, 1, 700.

Palmer, R. A., Newell, J. E., Gray, J., and Quinton, W. E. (1966). New England fournal of Medicine, 274, 248.

Quellhorst, E., Henning, H. V., and Scheler, F. (1973). Unpublished results.

Simkin, E. P., and Wright, F. K. (1968). Lancet, 1, 64.

Tenckhoff, H., and Curtis, F. K. (1970). Transactions of the American Society for Artificial Internal Organs, 16, 90.

Tenckhoff, H., and Schechter, H. (1968). Transactions of the American Society for Artificial Internal Organs, 14, 181. 